B. $2-68-28$

\title{
SOME SPECULATIVE $\mu$ EXPERIMENTS
}

Kwan Wu Lai

Brookhaven National Laboratory

\section{A Test of Lepton Quantum Numbers: Additive or Multplicative?}

In a weak or a electromagnetic process, $\mu-\operatorname{lepton}\left(1_{\mu}\right)$ and e - lepton $\left(1_{e}\right)$ numbers should separately be conserved. A process such as:

$$
\begin{aligned}
& \mu^{-} \mu^{-} \rightarrow e^{-} e^{-}, \\
& \begin{array}{lllll}
0 & 0 & -1 & -1 & 1
\end{array} \\
& +1+1 \quad 0 \quad 0 \quad 1_{\mu} \text {, }
\end{aligned}
$$

will be forbidden if the lepton quantum numbers are additive. However, this would be an allowed reaction if the lepton quantum numbers are multiplicative. A proposed test for this conservation law will be:

$$
\mu^{+} \mathrm{A} \rightarrow \mu^{-} \mathrm{e}^{+} \mathrm{e}^{+} \mathrm{A}
$$

and can be represented in a schematic diagram as follows:

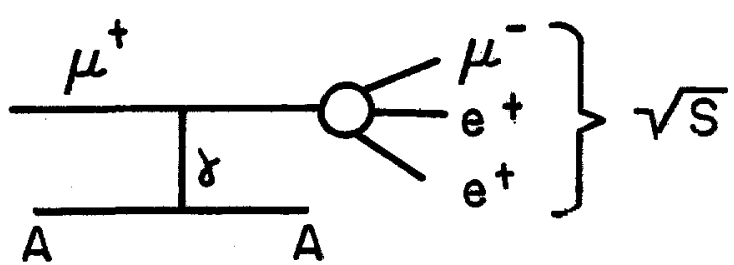

Fig. 1 
also

$$
\sqrt{S}=2 P_{\mu}\left(E_{\gamma}-P_{\gamma}\right)+q_{\gamma}^{2},
$$

where

$S$ is the total energy squared of the $\left(\mu^{-} e^{+} e^{+}\right)$system, $\mathrm{P}_{\mu}$ is the momentum of $\mu^{-}$,

$E_{\gamma}, P_{\gamma}$, and $q_{\gamma}^{2}$ are energy, 3-momentum, and 4-momentum of the virtual $\gamma$.

$A(Z)$ is a heavy nucleus.

If this process has a cross section of the order of $10^{-38} \mathrm{~cm}^{2}$ (competing with usual electromagnetic process of $10^{-30}$ to $10^{-33} \mathrm{~cm}^{2}$ ), detections of $\mu^{-}, e^{+}, e^{+}$in the final state would be difficult. However, if this process were manifest by a new interaction, then the cross section of this reaction could be as large as the usual electromagnetic process. One can also enhance the rate by an order of magnitude by means of the coherent process in a high-Z target, the experimental setup can be roughly shown below:

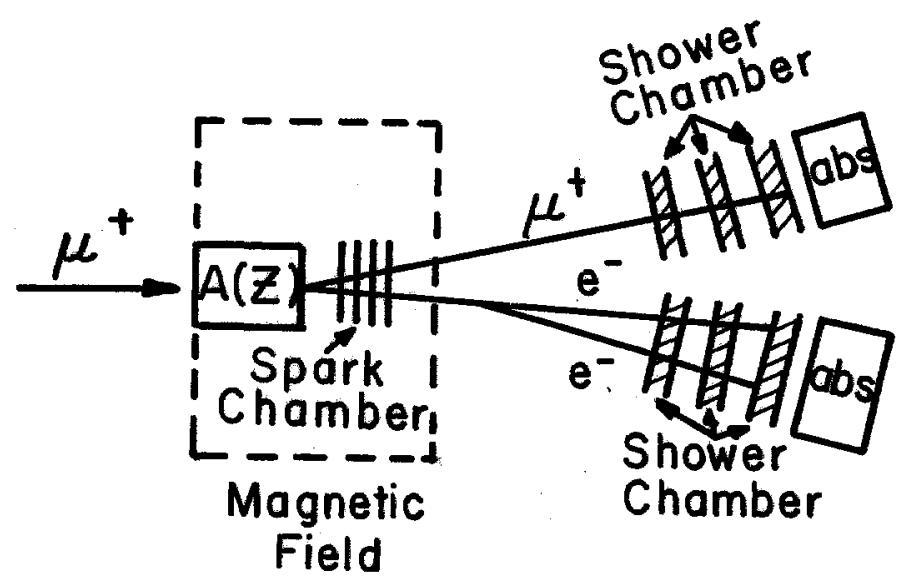

Fig. 2. 
Using shower chambers in both arms, one can also obtain events of the reaction

$$
\mu^{ \pm} \mathrm{A} \rightarrow \mu^{ \pm} \mathrm{e}^{+} \mathrm{e}^{-} \mathrm{A}
$$

This process should also provide a test for Q. E. D.

Search for Heavy Leptons of $\mu$-Type

One can use the missing-mass type of experiment with $\mu$ 's as the incident particles in the reaction (for example):

$$
\begin{aligned}
\mu^{ \pm} \mathrm{P} \rightarrow \mathrm{PX} & X^{ \pm} \\
& \bigsqcup_{\mu^{ \pm} \gamma},
\end{aligned}
$$

in a setup such as

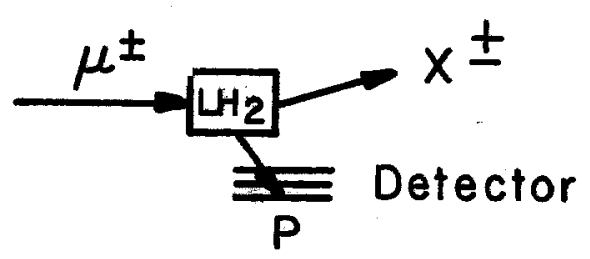

Fig. 3.

If the $X^{ \pm}$has a life time of $10^{-21}$ sec as suggested by $F$. Low for electron type of heavy leptons, then one can observe this object of a few $\mathrm{MeV}$ in width (comparable with the experimental resolution by selecting an appropriate Jacobian angle of the recoil protons). Figures 4 and 5 show kinematic plots of $\cos \theta_{p}$ vs $P_{p}$ in the lab system at incident $\mu^{ \pm}$momenta of 6 and $50 \mathrm{GeV} / \mathrm{c}$, with the $\mathrm{X}^{ \pm}$mass as parameter. 
Form Factor Study of Nucleon Isobars

Examine the form-factor behaviors of various known nucleon isobars and compare them with that of nucleons (double-pole fit to the form factor). In particular, the behavior of $\mathrm{P}_{11}$ nucleon isobar $\left[N^{*} 1 / 2(1400)\right]$ is of great interest in inelastic reactions, such as

$$
\stackrel{\mu \mathrm{P} \rightarrow \mu \mathrm{N}^{*}}{\longrightarrow}\left[\begin{array}{l}
\pi \mathrm{P} \\
\Lambda \mathrm{K},
\end{array}\right.
$$

at high energy as a function of 4-momentum transfer. 


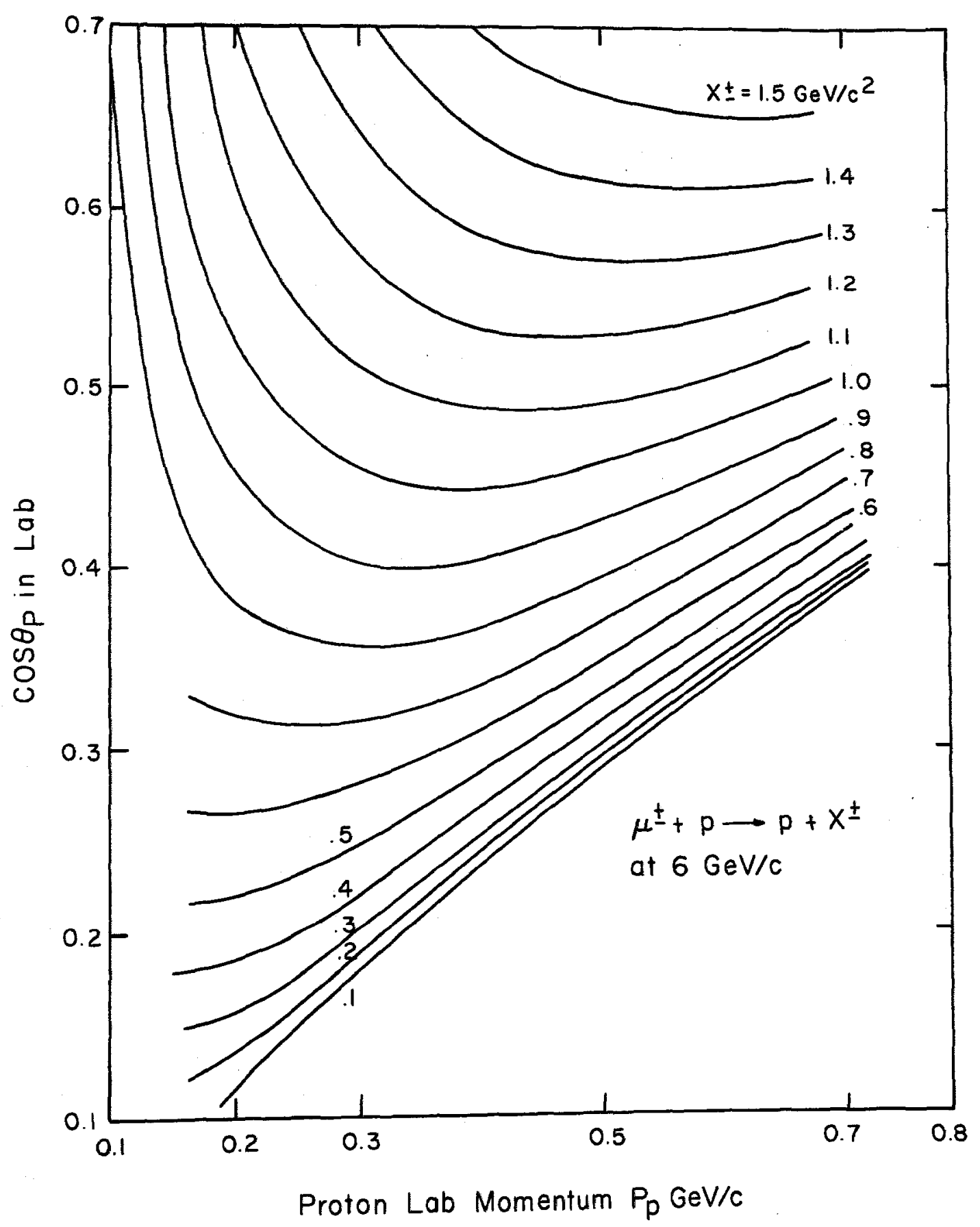

Fig. 4. Distribution in angle and momentum of secondaries from $\mathrm{mu}-\mathrm{p}$ interaction, as a function of the mass of the particle produced; primary momentum $6 \mathrm{GeV} / \mathrm{c}$. 


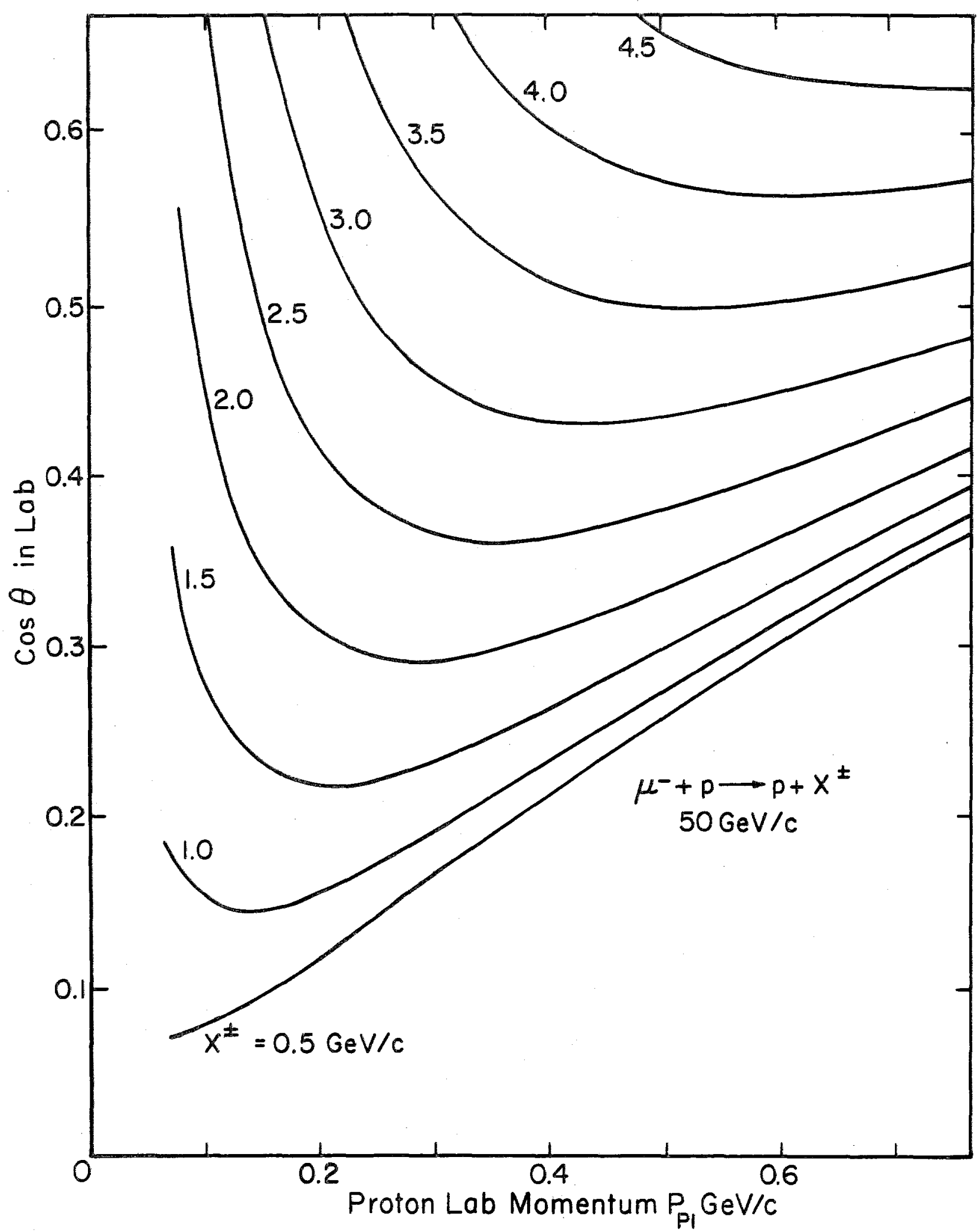

Fig. 5. Same as Fig. 4, for primary momentum $50 \mathrm{GeV} / \mathrm{c}$. 